\title{
Dynamic Signal Processing Based on Morphological Filter
}

\author{
YANG Haima ${ }^{1,2,3, a}$, MA Caiwen ${ }^{2, b}$, WANG Jianyu ${ }^{4, c}$ and LIU Jin ${ }^{3, d}$ \\ ${ }^{1}$ Xi'an Institute of Optics and Precision Mechanics, Xi'an 710119, China \\ ${ }^{2}$ University of Chinese Academy of Sciences, Beijing 100049, China \\ ${ }^{3}$ School of Optical-Electrical and Computer Engineering, USST, Shanghai 200093, China \\ ${ }^{4}$ Shanghai Institute of Technical Physics of the CAS, Shanghai, 200083, China \\ asnowyhm@sina.com, ' ${ }^{\mathrm{a}}$ Macaiwen2000@sina.com, 'Wangjianyu2013@sina.com, \\ dFlyingpine@sina.com
}

Keywords: Morphological filter, Structure element, Dynamic signal, Real-time control.

\begin{abstract}
In order to filter out all kinds of interference noises in batching control and improve the precision of the ingredients, a morphology filtering method was used in data processing which is the combination of morphological open-closing and close-opening. When the morphology filter is used to de-noise the interference noise, it doesn't need to consider the dynamic signal spectrum characteristics. After data processing and application in practical production, it is proved that the random noise, pulse noise and mixture noise can be removed effectively. The experiment shows that the simple and realized easily algorithm can be used in facilitate industrial fields and real-time signal processing.
\end{abstract}

\section{Introduction}

On the scene of mixing control, there exits various of peak pulse interference generated by the starting and stopping of high-power inductive loads, electromagnetic interference produced by relay and random noise created by the other equipments. These above noises have a serious affection when obtaining precise weighing signal. Many methods have been used in filtering noise in engineering fields, such as amplitude limiting filter, wavelet transformation and so on. And a good filtering effect have been achieved. However, with Amplitude limiting filter, the choice of the threshold value and corresponding replacement value of data points after removing pulse can produce a great deal of error and reduce the accuracy of the weighing signal. Wavelet transformation, when used in de-noising random noise, have a great deal of disadvantage such as band aliasing, phase shift and amplitude attenuation calculation amount .Taken everything into consideration, it is not suitable for acquisition of weighing signal on the scene of mixing control .For the special requirements of data collection and increasing accuracy on batching scene morphological filtering method is adopted in this paper, selecting the cosine structural elements. In this way, the noise in the weighing signal is eliminated, effectively, the accuracy of weighing signal in the process of the mixing control sene is also improved obviously.

\section{Principle of mathematical morphological filtering and it's construction}

\section{Mathematical morphological filtering principle}

Mathematical morphology is a filtering method to describe the input signal based on mathematics set theory, which is non-linear analytic method different from those based on time domain or frequency domain. Mathematical morphology is widely used in image processing, shape analysis, pattern recognition, power systems, computer vision and medical aspects.However, it is much less popular in the field of industrial control. Morphological filtering based on characteristics of the input signal collect information of the signal by selecting the probe of the appropriate collection of information, which is also known as "structural elements".It matches the signal by moving the 
structural elements in the input signal in this way,useful information of the signal can be extracted and noise can be suppressed.Noisy signal are often superimposed on the signal in the form of peak and the bottom , therefore, open - closing cascading combination should be used to eliminate noise in practice.

\section{Construction of mathematical morphological filtering}

The signal is one-dimensional signal in the mixing control scene,It is very important to design the morphological filter in this paper.The input signal that is $f(n)$ and the structure element is $g(n) . f(n)$ and $g(n)$ are both discrete function , and theirs definition domain are $F=\{0,1, \cdots, N-1\}$ and $G=\{0,1, \cdots, M-1\}$ and also the condition $N>M$ is required,. The basic morphological operations are erosion $\oplus$ and dilation $\Theta$, two other operations that derived from the basic ones are opening ${ }^{\circ}$ and closing $\bullet$, and theirs definition such as equation (1)and (2) .

$$
\begin{aligned}
& (f \circ g)(n)=[(f \Theta g) \oplus g](n) \\
& (f \bullet g)(n)=[(f \oplus g) \Theta g](n)
\end{aligned}
$$

So the basic filter can be designed such as equation(5)and (6) follows.

$$
\begin{aligned}
& O C(f(n))=(f \circ g \bullet g)(n) \\
& C O(f(n))=(f \bullet g \circ g)(n)
\end{aligned}
$$

$O C$ and $C O$ represent open-closing and close-opening.

Alternately mixed filter

$$
y(n)=\frac{[O C(f(n))+C O(f(n))]}{2}
$$

In the formula, $y(n)$ is output of morphological filter.

\section{Morphological filter applying to signal processing}

Ideally,the weighing signal is a linear in the mixing control system,however,it can be changed by the factor such as various noises , and interference which is engendered by the amplifier and regulate circuit ,meanwhile the quality is also effected.The signal that is obtained in the batching scene includes noises such as random noise,sinusoid noise which is engendered by control value and pulse noise that is generated by large power equipment and relay .In order to filter out the noises ,the type of cosine structure element is used to filter out the random noise,pulse noise,sinusoid noise which are superposed to the weighing signal in this paper.The result can be measured by root mean square and correlation coefficient ,the smaller the root mean square and the larger correlation coefficient is ,the better the result is. The equation as follows .

$$
\begin{aligned}
d & =\left[\frac{1}{N} * \sum_{n=1}^{N}(y(n)-f(n))^{2}\right]^{1 / 2} \\
R_{X Y} & =\frac{\sum_{i=1}^{N}\left(X_{i}-\bar{X}\right)\left(Y_{i}-\bar{Y}\right)}{\sqrt{\sum_{i=1}^{N}\left(X_{i}-\bar{X}\right)^{2}} \sqrt{\sum_{i=1}^{N}\left(Y_{i}-\bar{Y}\right)^{2}}}
\end{aligned}
$$

$X$ and $Y$ represent input signal $f(n)$ and output signal $y(n)$ 

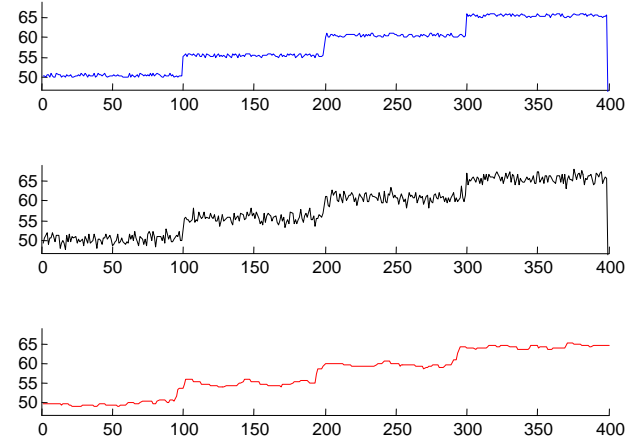

Figure 1 Superposed random noise
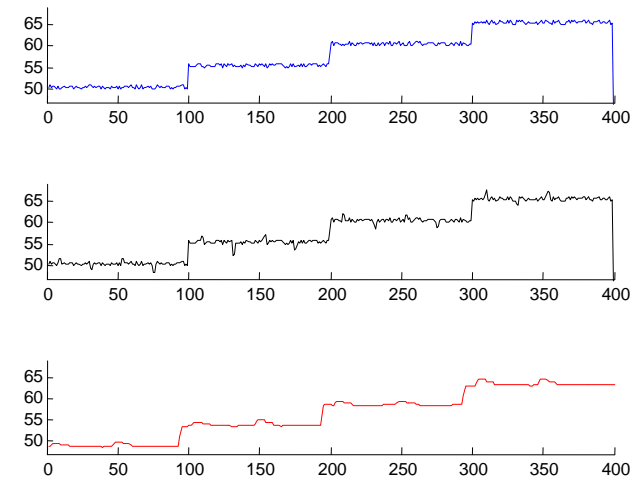

Figure 3 Superposed pulse noise
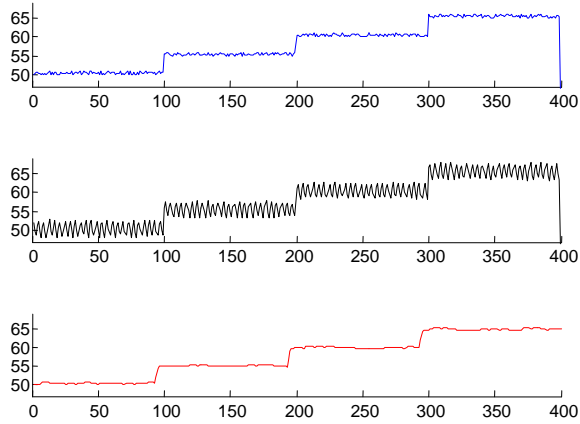

Figure 2 Superposed sinusoid noise
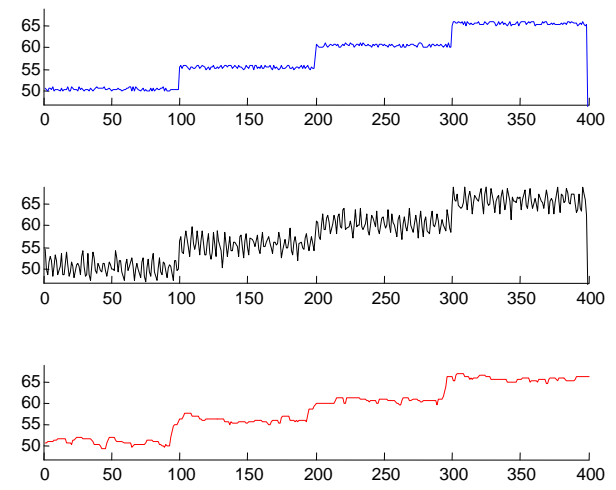

Figure 4 Superposed mixed noise

According to the figure 1,figure 2,figure 3,figure 4,the conclusion shows that:random noise ,sinusoid noise and pulse noise can be effectively removed, and the filtering result is ideal.The root mean square and correlation coefficient shown as table (1)and (2).

Table 1 The value of the root mean square

\begin{tabular}{|l|l|l|}
\hline Kinds of superposition & Before the filtering & After the filtering \\
\hline Random noise & 3.2768 & $5.2031 \mathrm{e}-26$ \\
\hline Sinusoid noise & 8.6192 & $1.7772 \mathrm{e}-26$ \\
\hline Pule noise & 0.3208 & $1.9117 \mathrm{e}-24$ \\
\hline Mixed noise & 3.2850 & $3.7328 \mathrm{e}-25$ \\
\hline
\end{tabular}

Table 2 The value of the correlation coefficient

\begin{tabular}{|l|l|}
\hline Kinds of superposition & correlation before and after filtering \\
\hline Random noise & $R=0.8408$ \\
\hline Sinusoid noise & $R=0.8352$ \\
\hline Pule noise & $R=0.8421$ \\
\hline Mixed noise & $R=0.8381$ \\
\hline
\end{tabular}

The conclusion can be found in the table 1and table 2,the weighing signal feature can be retained after filtering

\section{Summary}

Morphological filter is a kind of non-linear digital signal processing technology, it is widely used in two-dimensional image processing and shape analysis. The morphological filter is applied in the industrial control field in this paper,pulse noise and random noise can be removed effectively by constructing one filter that based on morphological opening and closing operation cascade combination,a new approach is provided for industrial field signal processing.Various noises can be effectively restrained with morphological filter , the result is much better than one of the methods 
such as limiting filtering, median filtering, moving filtering , and its processing speed and the simple algorithm are obviously superior to the commonly used wavelet transform .

\section{References}

[1] LI Bing-lin,WEI Min-xiang.Cylinder pressure signal processing in engine based on morphological filter[J].Transducer and Micro system Technologies ,2009,7(28):56-58.

[2] LIU Shu.Applying the mathematical morphology in signal processing[D].Dalian University of technology,2006.

[3] TANG Chang-qing,LV Hong-bo,HUANG Zheng.Mathematics Morphological Method and Its Application[M].Beijing:Science Press,1990.

[4] J. F. Zhang, and D. J. Zhang, S.Ultra-High-Speed Directional Protection of Transmission Lines Using Mathematical Morphology[J].IEEE Transactions on Power delivery,2003,18(4):1127-1133.

[5] Maragos P,Ronald W.S.Morphological Filters-Part I:Their Set-Theoretic Analysis and Relations to Linear Shift-Invariant Filters[J].IEEE Transactions on Acoustics Speech and Signal Processing,1987, 35(8):1153-1169.

[6] Maragos,Ronald W.S.Morphological Filters-Part II: Their relation to median,order statistic,and stack filter[J].IEEE Transactions on ASSP ,1987,35(8):1170-1184.

[7] Li Qing ,Wang Run-qi ,Huang Wen-feng and Zheng Gui-juan.Method for Morphological Filtering in Seismic Data Processing[J].Petroleum Science ,2005,2(4):20-29

[8] CHEN Ping,LI Qing-min.Design and analysis of mathematical morphological based digital filters [J].Processing of the CSEE ,2005,25(11):60-65.

[9] LI Chun-zhi,HE Rong-jian,TIAN Guang-ming.Research on the Application of the Mathematical Morphology Filtering in Vibration Signal Analysis[J].Computer Engineering\&Science, 2008,9(30): 126-127.

[10] ZHAO Zhao,LIU Li-lin,ZHANG Cheng-xue.Research and analysis of morphological filter's structure element selection principle[J].Power System Protection and Control,2009,14(37):21-25.

[11] OU Yang-shen,WANG Jian-wang,CHEN Dei-gui.A New Fast Disturbance Location Method Based On Mathematical Morphology For Power Quality Signal[J].Automation of Electric Power Systems,2003,19(27):25-29.

[12] LI Tian-yun,WANG Xue-qi,AN Bo.Application of Novel Generalized Morphological Filter to MOA On-line Monitoring Data Processing[J].Journal Of Northeast Dianli University Natural Science Edition,2009,4(29):45-49. 